\title{
联合对角化-DOA 矩阵方法
}

\author{
夏铁骑 ${ }^{*}$, 汪学刚, 郑毅, 万群 \\ 电子科技大学电子工程学院 706 教研室, 成都 610054 \\ *E-mail: yunguihun@163.com
}

收稿日期: 2007-04-13; 接受日期: 2007-07-13

国家自然科学基金(批准号: 60372022)和新世纪优秀人才支持计划(NCET-05-0806)资助项目

\begin{abstract}
摘要提出了一种新的基于联合对角化的高分辨二维 DOA 估计方法一 联合对角化-DOA 矩阵方法. 该方法在保持原 DOA 矩阵方法无需二维谱峰搜 索和参数配对等优点的基础上, 利用阵列的特殊结构构建 3 个子阵, 然后通 过联合对角化获得信号的二维角估计, 较好地解决了原 DOA 矩阵法存在的 角度兼并问题。仿真结果证明了该方法的有效性.

关键词

阵列天线

DOA

联合对角化

配对
\end{abstract}

在雷达、声纳、移动通信及谱分析等领域, 高性能的二维(2-D) DOA估计起着相当重要的 作用. 2-D DOA的特征结构算法, 如 2-D MUSIC类 ${ }^{[1]}$ 和 2-D ESPRIT类 ${ }^{[2,3]}$ 方法等, 都不可避免 地遇到诸如 2-D谱峰搜索、非线性优化、分维处理及参数配对等难题, 而且与一维(1-D)问题不 同, 2-D DOA估计必须借助平面阵或立体阵, 阵元数较多, 因此计算量、存它量大, 给算法的实 用性造成了不小的障碍. 殷勤业等 $[4,5]$ 利用双平线阵提出了DOA矩阵法, 比较有效地解决了上 述问题. 从理论上可以证明 ${ }^{[6]}$, 著名的ESPRIT方法只是DOA矩阵方法的特例. 然而, 不论是原 DOA矩阵法 ${ }^{[4,5]}$, 还是空时DOA矩阵法 ${ }^{[7,8]}, 2$-D ESPRIT方法 ${ }^{[2]}$, 都存在着角度兼并问题, 即不 能估计在某些平面或曲面内的信号的到达角，这降低了阵列的实用性. 尽管西 2-D ESPRIT ${ }^{[9]}$ 能够获得闭环形式的 2-D角估计, 对二维角实现自动配对, 且能够避免角兼并问题, 但其要求 阵列流形直接或者经过变化后具有中心共轭对称结构, 常见阵形有均匀矩形方阵 [9]、均匀圆阵 [1], 以及其他均匀中心对称阵 ${ }^{[10]}$. 若阵列不具有中心共轭对称结构时, 西 2-D ESPRIT算法很 难实现 $[9]$. 本文提出了一种基于联合对角化 (JD)的DOA矩阵方法, 利用阵列的特殊结构构建 3 个子阵, 不要求阵列流形具有中心共轭对称结构. 该方法较好地解决了存在角度兼并的信号 2-D DOA估计问题, 并保持了原DOA矩阵方法无需二维谱峰搜索和参数配对等优点. 最后给 出了计算机仿真结果.

\section{1 阵列结构及信号模型}

信号接收阵列为如图 1 所示的均匀平行 $\mathrm{L}$ 阵列, 阵列的总阵元数为 $2 K$ (其中, $K=M+N-3$ ), 
阵元之间的间距为 $d$. 假设空间有 $D$ 个非相关的窄带同载波信号 $s_{i}(t)(1 \leqslant i \leqslant D)$ 入射到此阵 列, 其与 $x$ 轴的夹角为 $\alpha_{i}$, 与 $y$ 轴的夹角为 $\beta_{i}$. 以阵列 $X$ 的第 1 阵元为参考点, 如图 1(b)所示, 把阵列分成 3 个子阵 $X_{a}, Y_{a}$ 和 $Z_{a}, t$ 时刻阵列对应的输出信号分别为

$$
\begin{gathered}
x(t)=\boldsymbol{A} \boldsymbol{s}(t)+\boldsymbol{n}_{x}(t), \\
y(t)=\boldsymbol{A} \boldsymbol{\Phi}_{1} \boldsymbol{s}(t)+\boldsymbol{n}_{y}(t), \\
z(t)=\boldsymbol{A} \boldsymbol{\Phi}_{2} \boldsymbol{s}(t)+\boldsymbol{n}_{z}(t),
\end{gathered}
$$

其中 $\boldsymbol{n}_{x}(t), \boldsymbol{n}_{y}(t)$ 和 $\boldsymbol{n}_{z}(t)$ 为 3 个子阵的加性白 Gauss 噪声向量, 其与信号 $\mathrm{s}(t)$ 相互独立. $\boldsymbol{x}=$ $\left[x_{1}(t), \cdots, x_{K}(t)\right]^{\mathrm{T}}, \quad \boldsymbol{y}=\left[y_{1}(t), \cdots, y_{K}(t)\right]^{\mathrm{T}}, \mathbf{z}=\left[z_{1}(t), \cdots, z_{K}(t)\right]^{\mathrm{T}}, \quad \boldsymbol{s}=\left[s_{1}(t), \cdots, s_{D}(t)\right]^{\mathrm{T}}, \quad \boldsymbol{A}=\left[\boldsymbol{a}_{1}, \cdots\right.$, $\left.\boldsymbol{a}_{i}, \cdots, \boldsymbol{a}_{D}\right]^{\mathrm{T}}$,

$$
\begin{aligned}
& \boldsymbol{a}_{i}=\exp \left\{\mathrm{j} 2 \pi \frac{d}{\lambda}\left[(N-2) \cos \left(\beta_{i}\right), \cdots, \cos \left(\beta_{i}\right), 0, \cos \left(\alpha_{i}\right), \cdots,(M-2) \cos \left(\alpha_{i}\right)\right]\right\}^{\mathrm{T}}, i=1, \cdots, D, \\
& \boldsymbol{\Phi}_{1}=\operatorname{diag}\left[\mathrm{e}^{\mathrm{j} \frac{2 \pi}{\lambda} d \cos \left(\beta_{1}\right)}, \cdots, \mathrm{e}^{\mathrm{j} \frac{2 \pi}{\lambda} d \cos \left(\beta_{D}\right)}\right]=\operatorname{diag}\left[\phi_{11}, \cdots, \phi_{p 1}, \cdots, \phi_{q 1} \cdots, \phi_{D 1}\right], \quad 1 \leqslant p \neq q \leqslant D, \\
& \boldsymbol{\Phi}_{2}=\operatorname{diag}\left[\mathrm{e}^{\mathrm{j} \frac{2 \pi}{\lambda} d \cos \left(\alpha_{1}\right)}, \cdots, \mathrm{e}^{\mathrm{j} \frac{2 \pi}{\lambda} d \cos \left(\alpha_{D}\right)}\right]=\operatorname{diag}\left[\phi_{12}, \cdots, \phi_{p 2}, \cdots, \phi_{q 2} \cdots, \phi_{D 2}\right], \quad 1 \leqslant p \neq q \leqslant D .
\end{aligned}
$$

可以看到 $(\alpha, \beta)$ 同时存在于方向矢量 $\boldsymbol{a}$ 中, 若能够估计出 $\boldsymbol{A}$, 则可以获得 $(\alpha, \beta)$ 的估计.
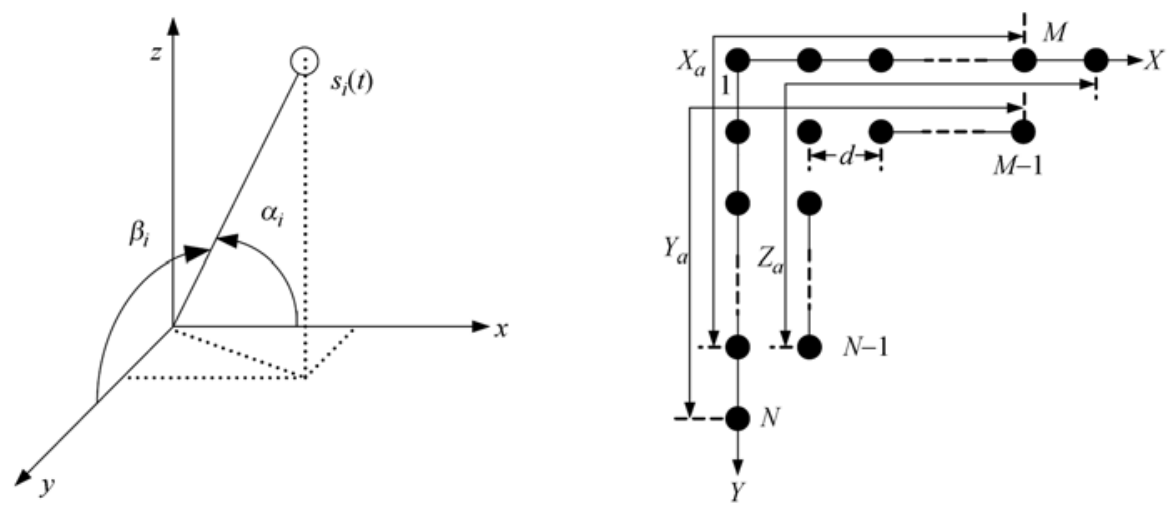

图 1 独立 2-D 角配置的阵列结构图

(a) 独立 2-D 角配置关系图; (b) 阵列平面结构图

接收到的数据 x $(t)$ 的自相关矩阵为 $\boldsymbol{R}_{x x}$, 则有

$$
\boldsymbol{R}_{x x}=E\left[\boldsymbol{x}(t) \boldsymbol{x}(t)^{\mathrm{H}}\right]=\boldsymbol{A} \boldsymbol{P} \boldsymbol{A}^{\mathrm{H}}+\sigma^{2} \boldsymbol{I},
$$

其中 $\boldsymbol{P}=E\left[\boldsymbol{s}(t) \boldsymbol{s}(t)^{\mathrm{H}}\right]$, 为信号源的协方差矩阵, $\boldsymbol{I}$ 为 $K \times K$ 维的单位矩阵. $\sigma^{2}$ 为加性白 Gauss 噪声的方差. 上标 $\mathrm{H}$ 表示复共轭转置.

$\boldsymbol{y}(t)$ 和 $\boldsymbol{x}(t)$ 的互相关矩阵为 $\boldsymbol{R}_{y x}$, 


$$
\boldsymbol{R}_{y x}=E\left[\boldsymbol{y}(t) \boldsymbol{x}(t)^{\mathrm{H}}\right]=\boldsymbol{A} \boldsymbol{\Phi} \boldsymbol{P} \boldsymbol{A}^{\mathrm{H}}+\sigma^{2} \Sigma_{d},
$$

其中 $\sum_{d}$ 为 $K \times K$ 的矩阵, $\boldsymbol{J}_{d}$ 为 $(N-1) \times(N-1)$ 的矩阵,

$$
\Sigma_{d}=\left[\begin{array}{cc}
\boldsymbol{J}_{d} & \mathbf{0} \\
\mathbf{0} & \mathbf{0}
\end{array}\right], \quad \boldsymbol{J}_{d}=\left[\begin{array}{ccccc}
0 & 0 & \cdots & \cdots & 0 \\
1 & 0 & \cdots & \cdots & 0 \\
0 & 1 & \ddots & \ddots & 0 \\
\vdots & \ddots & \ddots & \ddots & \vdots \\
0 & \cdots & 0 & 1 & 0
\end{array}\right] .
$$

$\boldsymbol{z}(t)$ 和 $\boldsymbol{x}(t)$ 的互相关矩阵为 $\boldsymbol{R}_{z x}$,

$$
\boldsymbol{R}_{z x}=E\left[\mathbf{z}(t) \boldsymbol{x}(t)^{\mathrm{H}}\right]=\boldsymbol{A} \boldsymbol{\Phi}_{2} \boldsymbol{P} \boldsymbol{A}^{\mathrm{H}}+\sigma^{2} \Sigma_{e},
$$

其中 $\Sigma_{e}$ 为 $K \times K$ 的矩阵, $\boldsymbol{J}_{e}$ 为 $(M-1) \times(M-1)$ 的矩阵,

$$
\Sigma_{e}=\left[\begin{array}{cc}
\mathbf{0} & 0 \\
\mathbf{0} & \boldsymbol{J}_{e}
\end{array}\right], \quad \boldsymbol{J}_{e}=\left[\begin{array}{ccccc}
0 & 1 & 0 & \cdots & 0 \\
0 & 0 & 1 & \ddots & \vdots \\
0 & 0 & \ddots & \ddots & 0 \\
\vdots & \ddots & \ddots & \ddots & 1 \\
0 & \cdots & \cdots & 0 & 0
\end{array}\right] .
$$

对 $\boldsymbol{R}_{x x}$ 进行特征值分解 $(\mathrm{EVD})$, 令 $\lambda_{1}, \cdots, \lambda_{D}$ 为矩阵 $\boldsymbol{R}_{x x}$ 的 $D$ 个大特征值, $\boldsymbol{h}_{1}, \cdots, \boldsymbol{h}_{D}$ 为其对应 的特征向量, 在白噪声的假设下, 可以由 $K-D$ 个小特征值的平均得到噪声方差 $\sigma^{2}$ 的估计, 然后, 通过去除噪声的影响, 可以得到 $\boldsymbol{R}_{x x}, \boldsymbol{R}_{y x}$ 和 $\boldsymbol{R}_{z x}$ 的无噪方差矩阵, 即

$$
\begin{gathered}
\boldsymbol{C}_{x x}=\boldsymbol{A} \boldsymbol{P} \boldsymbol{A}^{\mathrm{H}}=\boldsymbol{R}_{x x}-\sigma^{2} \boldsymbol{I}, \\
\boldsymbol{C}_{y x}=\boldsymbol{A} \boldsymbol{\Phi} \boldsymbol{P A}^{\mathrm{H}}=\boldsymbol{R}_{y x}-\sigma^{2} \Sigma_{d}, \\
\boldsymbol{C}_{z x}=\boldsymbol{A} \boldsymbol{\Phi}_{2} \boldsymbol{P} \boldsymbol{A}^{\mathrm{H}}=\boldsymbol{R}_{z x}-\sigma^{2} \Sigma_{e} .
\end{gathered}
$$

$C_{x x}$ 的伪逆由(13)式给出,

$$
\boldsymbol{C}_{x x}^{\dagger}=\sum_{i=1}^{D} \lambda_{i}^{-1} \boldsymbol{h}_{i} \boldsymbol{h}_{i}^{\mathrm{H}},
$$

根据 DOA 矩阵方法的思想, 可以分别得到如下的 DOA 矩阵:

$$
\begin{aligned}
& \boldsymbol{R}_{y}=C_{y x} C_{x x}^{\dagger}, \\
& \boldsymbol{R}_{z}=C_{z x} C_{x x}^{\dagger} .
\end{aligned}
$$

定理 1 如果 $\boldsymbol{A}$ 与 $\boldsymbol{P}$ 满秩, $\boldsymbol{\Phi}_{1}$ 或 $\boldsymbol{\Phi}_{2}$ 无相同的对角元素, 则 DOA 矩阵的 $D$ 个非零特征值 等于 $\boldsymbol{\Phi}_{1}$ 或 $\boldsymbol{\Phi}_{2}$ 中 $D$ 个对角元素, 而这些值对应的特征向量等于相应的信号方向矢量, 即

$$
\begin{aligned}
& R_{y} A=A \Phi_{1}, \\
& R_{z} A=A \Phi_{2} .
\end{aligned}
$$

证明见文献 [6]. 因此只要对 $D O A$ 矩阵 $\boldsymbol{R}_{y}$ 或 $\boldsymbol{R}_{z}$ 进行特征分解, 就可以直接得到矩阵 $\boldsymbol{A}, \boldsymbol{\Phi}_{1}$ 或 
$\boldsymbol{\Phi}_{2}$, 进而通过(4) (6)式求出 2-D DOA, 这种方法称为DOA矩阵方法. 采用(16)式估计 2-D DOA时, 要求 $\boldsymbol{\Phi}_{1}$ 不能具有相同的对角元素, 采用(17)式估计 2-D DOA时, 要求 $\boldsymbol{\Phi}_{2}$ 不能具有相 同的对角元素. 然而，在实际应用中，接收到的信号常常部分具有相同或间距很小的 $\alpha$, 另一 部分具有相同或间距很小的 $\beta$, 此时这里的DOA矩阵方法将不能得到正确的DOA估计.

定理 1 成立的关键条件是 $\boldsymbol{\Phi}_{1}$ 和 $\boldsymbol{\Phi}_{2}$ 均无相同的对角元素, 然而在某些信号具有相同的 $\beta$ 角度时, $\boldsymbol{\Phi}_{1}$ 将具有相同的元素, 在另一些信号具有相同的 $\alpha$ 角度时, $\boldsymbol{\Phi}_{2}$ 将具有相同的元素, 因此导致原 DOA矩阵法失效. 但是, 一般假设信号 $s_{p}(t)$ 和 $s_{q}(t)(1 \leqslant p \neq q \leqslant D)$ 只能具有相同 的一维角, 因此可以通过找到一种综合两个 DOA 矩阵 $\boldsymbol{R}_{y}$ 和 $\boldsymbol{R}_{z}$ 中的 DOA 信息的方法, 进行二 维 DOA 估计, 即下节的 JD-DOA 矩阵方法.

\section{JD-DOA 矩阵方法的基本原理}

在进行 JD 之前, 我们先进行如下的假设:

$$
\boldsymbol{x}(t)=\boldsymbol{A} \boldsymbol{s}(t)+\boldsymbol{n}_{x}(t)=\sum_{i=1}^{D} \frac{\boldsymbol{a}_{i}}{c_{i}} c_{i} s_{i}(t)+\boldsymbol{n}_{x}(t),
$$

其中 $c_{i}$ 为一个任意的复因子. 不失一般性, 可以假设 $\boldsymbol{P}=\boldsymbol{I}$, 而把第 $i$ 个信号源的幅度归入 $\boldsymbol{a}_{i}$ 中. 这种归一化处理将简化下面的推导, 但不影响 2-D DOA 的估计性能.

定义 1 矩阵 $\boldsymbol{B}$ 和 $\boldsymbol{E}$ 本质相等, 若存在一个矩阵 $\boldsymbol{F}$, 使得 $\boldsymbol{B}=\boldsymbol{E} \boldsymbol{F}$, 其中 $\boldsymbol{F}$ 的每一行和每 一列中只有一个非零元素, 其模为 1 . 记为 $\boldsymbol{B} \doteq \boldsymbol{E}$.

\section{1 白化降维处理}

JD-DOA 矩阵方法的第 1 步是对 $\boldsymbol{R}_{x x}$ 进行特征值分解, 得到一个白化矩阵 $\boldsymbol{W}$,

$$
\boldsymbol{W}=\left[\left(\lambda_{1}-\sigma^{2}\right)^{-1 / 2} \boldsymbol{h}_{1}, \cdots,\left(\lambda_{D}-\sigma^{2}\right)^{-1 / 2} \boldsymbol{h}_{D}\right]^{\mathrm{H}},
$$

使得

$$
E\left[\boldsymbol{W} \boldsymbol{R}_{x x} \boldsymbol{W}^{\mathrm{H}}\right]=\boldsymbol{W} \boldsymbol{A} \boldsymbol{A}^{\mathrm{H}} \boldsymbol{W}^{\mathrm{H}}=\boldsymbol{I} .
$$

(20)式表明若 $W$ 为一个白化阵, 则 $W A$ 是一个 $D \times D$ 的西矩阵. 即对任意的白化矩阵 $\boldsymbol{W}$, 存在 一个 $D \times D$ 的西矩阵 $\boldsymbol{U}$, 使得 $\boldsymbol{W A}=\boldsymbol{U}$. 也就是说, 矩阵 $\boldsymbol{A}$ 可以分解为

$$
\boldsymbol{A}=\boldsymbol{W}^{\dagger} \boldsymbol{U} .
$$

这使得原问题由确定一个 $K \times D$ 的矩阵 $\boldsymbol{A}$ 变成确定一个 $D \times D$ 矩阵 $\boldsymbol{U}$, 从而降低了计算量.

对 $C_{y x}$ 和 $C_{z x}$ 进行白化降维处理, 得到

$$
\begin{gathered}
\boldsymbol{G}_{y x}=\boldsymbol{W} \boldsymbol{C}_{y x} \boldsymbol{W}^{\mathrm{H}}=\boldsymbol{U} \boldsymbol{\Phi}_{1} \boldsymbol{U}^{\mathrm{H}}, \\
\boldsymbol{G}_{z x}=\boldsymbol{W} \boldsymbol{C}_{z x} \boldsymbol{W}^{\mathrm{H}}=\boldsymbol{U} \boldsymbol{\Phi}_{2} \boldsymbol{U}^{\mathrm{H}} .
\end{gathered}
$$

\section{2 联合对角化}

JD-DOA 矩阵方法的第 2 步是估计联合对角化器 $\boldsymbol{U}$. 如果 $\boldsymbol{G}_{y x}=\boldsymbol{U} \boldsymbol{\Phi}_{1} \boldsymbol{U}^{\mathrm{H}}, \boldsymbol{G}_{z x}=\boldsymbol{U} \boldsymbol{\Phi}_{2} \boldsymbol{U}^{\mathrm{H}}$, 
则其只能被与 $\boldsymbol{U}$ 本质上相等的联合对角化器对角化, 即存在 $\boldsymbol{V} \doteq \boldsymbol{U}$, 使得

$$
L(\tilde{\boldsymbol{G}}, \boldsymbol{V}) \stackrel{\operatorname{def}}{=} \sum_{l=1}^{L} \operatorname{off}\left(\boldsymbol{V}^{\mathrm{H}} \boldsymbol{G}_{l} \boldsymbol{V}\right), \quad \boldsymbol{G}_{1}=\boldsymbol{G}_{y x}, \quad \boldsymbol{G}_{2}=\boldsymbol{G}_{z x},
$$

其中 $\operatorname{off}(\boldsymbol{M})$ 表示矩阵 $\boldsymbol{M}$ 的非主对角元素的绝对值平方总和.

定理 2 联合对角化的本质唯一条件: 令 $\tilde{\boldsymbol{G}}=\left\{\boldsymbol{G}_{y x}, \boldsymbol{G}_{z x}\right\}$ 为两个矩阵的集合, 对于 $l=1,2$, 矩阵 $\boldsymbol{G}_{y x}$ 和 $\boldsymbol{G}_{z x}$ 都可以表示成 $\boldsymbol{U} \boldsymbol{\Phi} \boldsymbol{U}^{\mathrm{H}}$ 的形式, $\boldsymbol{U}$ 是一个西矩阵. 任何 $\tilde{\boldsymbol{G}}$ 的联合对角化器与 $\boldsymbol{U}$ 本质上相等当且仅当

$$
\forall 1 \leqslant p \neq q \leqslant D, \quad \exists l, l=1,2, \quad \phi_{p l} \neq \phi_{q l} .
$$

本质唯一条件显然比定理 1 中对 $\boldsymbol{\Phi}_{1}$ 和 $\boldsymbol{\Phi}_{2}$ 的要求弱得多. 特别地, 很容易构造一些特例, 使得 $\boldsymbol{\Phi}_{1}$ 和 $\boldsymbol{\Phi}_{2}$ 都存在退化特征值谱, 然而却存在唯一的非退化的联合对角化器 $\boldsymbol{U}$. 定理 2 的 证明与文献 [11]中的证明类似.

定理 3 设 $\left(\alpha_{k}, \beta_{k}\right) \in[0, \pi) \times[0, \pi)$, 对于 $\boldsymbol{\Phi}_{1}$ 和 $\boldsymbol{\Phi}_{2}$, 存在联合对角化器 $\boldsymbol{U}$, 即

$$
\forall 1 \leqslant p \neq q \leqslant D, \quad \exists \phi_{p l} \neq \phi_{q l}, l=1,2 .
$$

证明 假设 $\left(\alpha_{i}, \beta_{i}\right) \in[0, \pi) \times[0, \pi)$, 对于第 $p$ 个信号的二维角 $\left(\alpha_{p}, \beta_{p}\right)$ 和第 $q$ 个信号源的二 维角 $\left(\alpha_{q}, \beta_{q}\right)(p \neq q)$, 存在下面 3 种情形:

1) 若 $\beta_{p} \neq \beta_{q}, \alpha_{p}=\alpha_{q}$, 则 $\phi_{p 1} \neq \phi_{q 1}$ 成立;

2) 若 $\beta_{p} \neq \beta_{q}, \alpha_{p} \neq \alpha_{q}$, 则 $\phi_{p 1} \neq \phi_{q 1}$ 和 $\phi_{p 2} \neq \phi_{q 2}$ 均成立;

3) 若 $\beta_{p}=\beta_{q}$, 那么 $\alpha_{p} \neq \alpha_{q}$, 则 $\phi_{p 2} \neq \phi_{q 2}$ 成立;

可见, 至少存在一个 $l(l=1,2)$, 满足 $\phi_{p l} \neq \phi_{q l}$. 然后通过(21)式估计 $\boldsymbol{A}$.

\subsection{JD-DOA 矩阵方法}

根据上面各节的讨论, 采用联合对角化技术实现了综合包含在两个 DOA 矩阵 $\boldsymbol{R}_{y}$ 和 $\boldsymbol{R}_{z}$ 中的 DOA 信息的目的, 这种方法称为 JD-DOA 矩阵方法. 下面给出 JD-DOA 矩阵方法的具体步骤:

1) 根据(7) (9)式分别估计 3 个子阵输出数据的采样协方差阵 $\boldsymbol{R}_{x x}, \boldsymbol{R}_{y x}$ 和 $\boldsymbol{R}_{z x}$;

2) 对 $\boldsymbol{R}_{x x}$ 进行特征值分解, 得到一个白化矩阵 $\boldsymbol{W}$;

3) 由(11)和(12)式去除噪声的影响, 可以得到的无噪方差矩阵 $\boldsymbol{C}_{y x}$ 和 $\boldsymbol{C}_{z x}$;

4) 通过(22)和(23)式对 $\boldsymbol{C}_{y x}$ 和 $\boldsymbol{C}_{z x}$ 分别进行白化降维处理, 得到 $\boldsymbol{G}_{y x}$ 和 $\boldsymbol{G}_{z x}$;

5) 对集合 $\left\{\boldsymbol{G}_{y x}, \boldsymbol{G}_{z x}\right\}$ 进行联合对角化得到 $\boldsymbol{U}$;

6) 矩阵 $\boldsymbol{A}$ 可以由 $\boldsymbol{A}=\boldsymbol{W}^{\dagger} \boldsymbol{U}$ 得到, 通过(4)式获得 2-D DOA 估计.

\section{3 计算复杂度分析}

一般情形下, 阵列接收的快摄数 $T$ 远大于天线数目, 即 $T \gg K$. 形成采样相关矩阵需要复 
乘数为 $K^{2} T$, 对一个 $K \times K$ 维的复矩阵进行特征分解需要复乘数为 $\left.O(K)^{3}\right)^{[12,13]}$. 因而DOA矩阵 法的所需复乘数约为 $\left(2 K^{2} T+O\left(K^{3}\right)\right)$, 本文方法所需复乘数约为 $\left(3 K^{2} T+O\left(K^{3}\right)\right)$, 可见JD-DOA矩 阵法的计算量约为原DOA矩阵法的 1.5 倍. 对于西 2-D ESPRIT方法, 其主要计算量在于计算 $2 K \times 2 T$ 维实矩阵的奇异值分解 ${ }^{[10]}$, 其计算量与原 $\mathrm{DOA}$ 矩阵法基本相当.

\section{4 计算机模拟及算法的精度比较和分析}

本节给出JD-DOA矩阵方法的计算机仿真结果. 在文献 [4]中, 已经对原DOA矩阵方法和 2-D MUSIC方法的性能进行了仿真和比较. 此处, 仅对JD-DOA矩阵方法和原DOA矩阵方法以 及西 2-D ESPRIT方法进行比较. 在性能仿真中, 信号估计的RMSE定义为

$$
\operatorname{RMSE}\left(\alpha_{i}, \beta_{i}\right)=\sqrt{E\left[\left(\hat{\alpha}_{i}-\alpha_{i}\right)^{2}+\left(\hat{\beta}_{i}-\beta_{i}\right)^{2}\right]} .
$$

仿真 1 无角兼并的信号源估计性能，比较JD-DOA矩阵方法和原DOA矩阵方法 ${ }^{[4]}$.

设 3 个不相关窄带信号源的 $\mathrm{DOA}$ 参数分别为 $\left(60^{\circ}, 60^{\circ}\right),\left(70^{\circ}, 76^{\circ}\right)$ 和 $\left(80^{\circ}, 80^{\circ}\right)$. 在 JD-DOA 矩阵方法中, 取 $N=3, M=8$, 即阵元总数为 16 . 为便于比较, 双平行阵中采用同样 的阵元数, 此时两种算法的阵列结构仅有一个阵元位置不同. $d=\lambda / 2$, 阵列接收的快摄数为 100. 图 2 为 3 个信号的均方根误差(RMSE)随信噪比(SNR)变化的性能曲线, 由 500 次 MonteCarlo 仿真取平均获得. 从图 2 中可以看出, $s_{1}(t)$ 与 $s_{2}(t)$ 和 $s_{3}(t) 2-\mathrm{D}$ 角间距均较大, 此时 JD$D O A$ 矩阵方法和原 DOA 矩阵方法对 $s_{1}(t)$ 具有基本一致的估计性能. 由于 $s_{2}(t)$ 和 $s_{3}(t)$ 的 $\beta$ 角 间距较小, JD-DOA 矩阵方法对 $s_{2}(t)$ 和 $s_{3}(t)$ 的估计性能明显优于原 DOA 矩阵方法, 改善了阵 列的角估计性能.
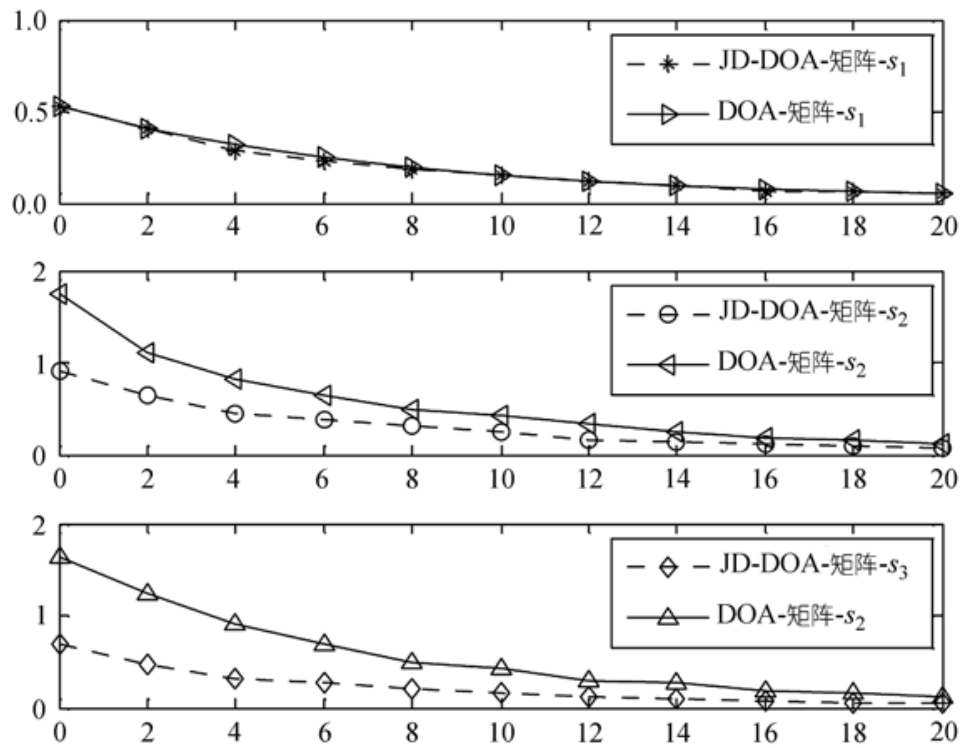

图 2 三个信号估计的均方根误差随信噪比变化的性能曲线 
仿真 2 存在角兼并的源信号估计, 比较JD-DOA矩阵方法和原DOA矩阵方法 ${ }^{[4]}$.

针对仿真 1 中的天线参数不变. 设 3 个信号的参数分别为 $\left(40^{\circ}, 50^{\circ}\right),\left(40^{\circ}, 70^{\circ}\right)$ 和 $\left(60^{\circ}, 70^{\circ}\right)$. 阵列接收的快摄数为 100 . 进行 50 次独立 Monte-Carlo 实验, 图 3(a) 和 3(b) 分别为 $\mathrm{SNR}=5 \mathrm{~dB}$ 时 JD-DOA 矩阵方法和原 $\mathrm{DOA}$ 矩阵方法的估计结果, 图 3(c)和 3(d) 分别为 $\mathrm{SNR}=$ $15 \mathrm{~dB}$ 时 JD-DOA矩阵方法和原 DOA 矩阵方法的估计结果. 可以看出, 入射信号 $s_{1}(t)$ 和 $s_{2}(t)$ 的 $\alpha$ 角相同, $s_{2}(t)$ 和 $s_{3}(t)$ 的 $\beta$ 角相同, 原 DOA 矩阵方法已经失效, 而 JD-DOA 矩阵法能够获得 较好的估计.
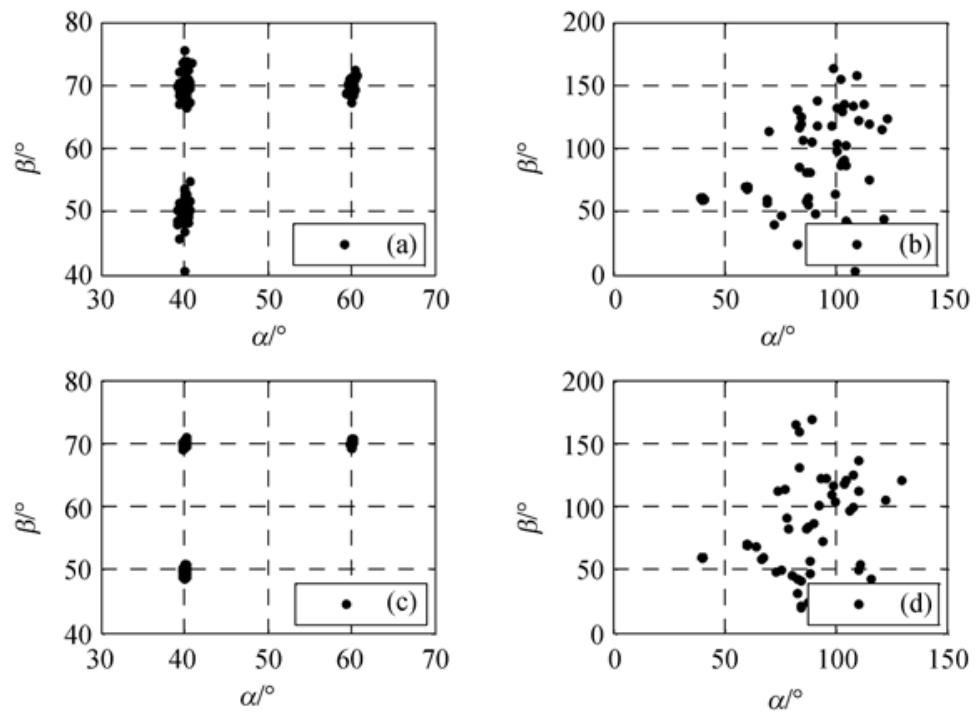

图 33 个信号的估计结果

(a)JD-DOA 矩阵方法, SNR=5dB; (b) 原 DOA 矩阵方法, SNR=5dB; (c)JD-DOA 矩阵方法, SNR=15dB; (d) 原 DOA 矩阵方 法, $\mathrm{SNR}=15 \mathrm{~dB}$

仿真 3 存在角兼并的源信号估计, 比较JD-DOA矩阵方法和西 2-D ESPRIT ${ }^{[9]}$ 方法.

设 3 个信号的参数分别为 $\left(50^{\circ}, 55^{\circ}\right),\left(50^{\circ}, 70^{\circ}\right)$ 和 $\left(65^{\circ}, 70^{\circ}\right)$. 可以看出, 入射信号 $s_{1}(t)$ 和 $s_{2}(t)$ 的 $\alpha$ 角相同, $s_{2}(t)$ 和 $s_{3}(t)$ 的 $\beta$ 角相同. 在 JD-DOA 矩阵方法中, 取 $N=6, M=7$, 即阵 元总数为 20 . 对于西 2-D ESPRIT 方法, 取为具有相同阵元数的 $5 \times 4$ 的矩形阵列, 此时两种算 法的阵列结构差别很大. $d=\lambda / 2$, 阵列接收的快摄数为 100 . 图 4 为 3 个信号的均方根误差 (RMSE) 随信噪比(SNR)变化的性能曲线, 由 500 次 Monte-Carlo 仿真取平均获得. 从图 4 可以 看出, 两种方法的估计性能基本一致, 在低信噪比下, 本文方法优于西 2-D ESPRIT 方法. 可 见, 对于不具有中心共轭对阵结构的阵列流形, JD-DOA 矩阵方法能够获得较好的估计性能.

\section{5 结论}

本文提出了一种新的基于联合对角化技术的高分辨二维 DOA 估计方法一JD-DOA 矩阵 方法. 该方法不仅较好地解决了存在角度兼并时窄带非相关信号 2-D DOA 估计问题, 还保持 了原 DOA 矩阵方法无需二维谱峰搜索和参数配对等优点, 计算量和原算法基本相当. 其最多 

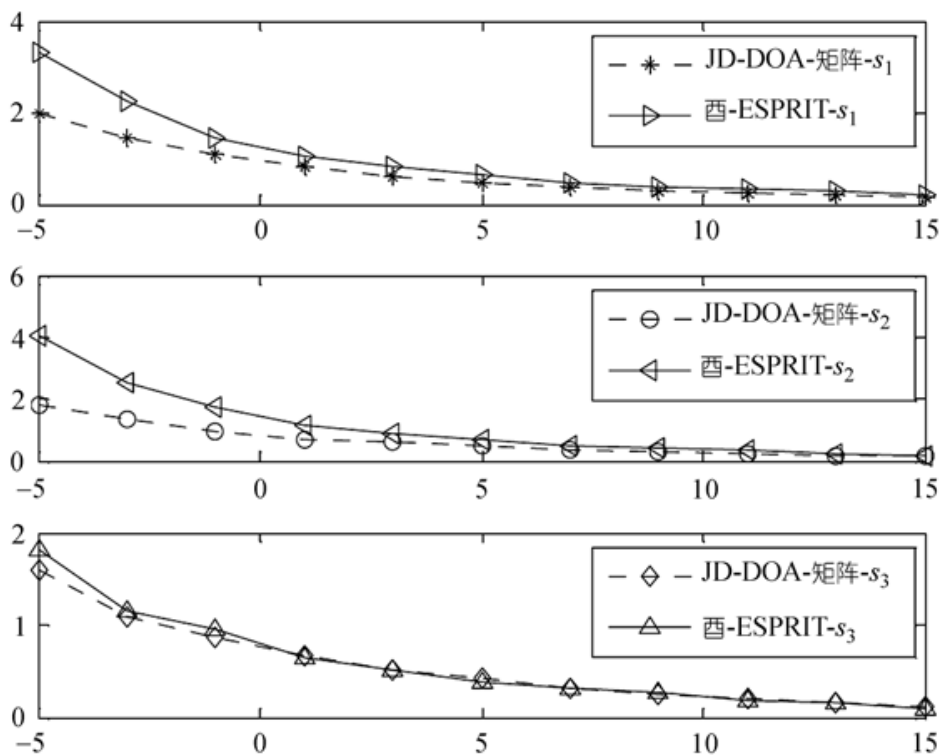

图 43 个信号估计的均方根误差随信噪比变化的性能曲线

可以估计 $K$ 个信号源的 2-D DOA. 在不存在 1-D 角兼并的情形下, 估计性能和原 DOA 矩阵方 法基本一致，存在 1-D 角兼并或者 1-D 角间距较小时，改善了阵列的角估计性能.

\section{参考文献}

1 Chan A Y J, Litva J. MUSIC and maximum likelihood techniques on two-dimensional DOA estimation with uniform circular array. In: IEE Proceedings of Radar, Sonar and Navigation, vol 142(3). New York: IEEE Press, 1995. 105-114

2 Kedia V S, Chandna B. A new algorithm for 2-D DOA estimation. Signal Process, 1997, 60(3): 325-332 [DOI]

3 van der Veen A J, Ober P B, Deprettere E F. Azimuth and elevation computation in high resolution DOA estimation. IEEE Trans Signal Process, 1992, 7(40): 1828 - 1832 [DOI]

4 殷勤业,邹理和, Newcomb R. 一种高分辨率二维信号参数估计方法——波达方向矩阵法. 通信学报, 1991, 4(12): $1-6$

5 Yin Q Y, Newcomb R, Zou L. Estimating 2-D angle of arrival via two parallel linear array. In: Proceedings of IEEE International Conference on Acoustic, Speech and Signal Processing, vol 3. New York: IEEE Press, 1989. $2803-2806$

6 殷勤业。高分辨率波达方向估计.博士学位论文. 西安: 西安交通大学

7 金梁, 殷勤业. 时空 DOA 矩阵方法. 电子学报, 2000, 7(28): 8-12

8 金梁, 殷勤业. 时空 DOA 矩阵方法的分析和推广。电子学报, 2001, 3(39): 300-303

9 Zoltowski M D, Haardt M, Mathews C P. Closed-form 2-D angle estimation with rectangular arrays in element space or beamspace via unitary ESPRIT. IEEE Trans Signal Processing, 1996, 2(44): 316-322 [DOI]

10 斯德谊, 乐强, 沈士团, 等. 用均匀中心对称阵二维酉 ESPRIT 方法实现闭环形式二维角估计. 电子学报, 1999, 3(27): 67-70

11 Belouchrani A, Abed-Meraim K, Cardoso J F, et al. A blind source separation technique using second-order statistics. IEEE Trans Signal Process, 1997, 2(45): 434-444 [DOI]

12 Roy R, Kailath T. ESPRIT-Estimation of signal parameters via rotational invariance techniques. IEEE Trans Acoustics Speech Signal Process, 1989, 7(37): 984-995 [DOI]

13 Marcos S, Marsal A, Benidir M. The propagator method for source bearing estimation. Signal Process, 1995, (42): $121-138$ 